\title{
A Bivariate Mathematical Model by Using Gonadotropin Releasing Hormone agonist (GnRHa) for the Prevention of Chemotherapy- Induced Ovarian Failure in Patients
}

\author{
Dr. S. Lakshmi ${ }^{1}$, Akanksha A. Desai ${ }^{2}$

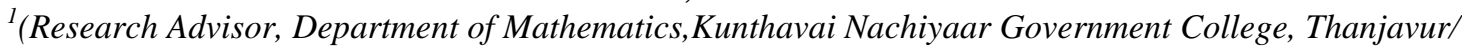 \\ Bharathidasan University, India) \\ ${ }^{2}$ (Research Scholar/ Bharathidasan University, India)
}

\begin{abstract}
The problem of generating bivariate life distributions from univariate once is drawing the attention of the reliability analyst. Amongst those approaches, the characterization approach and the modeling approach are very appealing. In fact characterization approach is of interest to both theoreticians and applied workers. Here we have used a bivariate distribution for application by extending the univariate distribution through characterization approach. In our application we have considered cancer patients with chemotherapy treatment and taking GnRHa, FSH, and Estradiol as women stress effects. .
\end{abstract}

Keywords: GnRHa, FSH, Estradiol

\section{Introduction}

Although cancer incidence rates in women less than 50years old continue to increase during recent years, mortality rates are dramatically decreasing due to modern advances in treatment. Modern techniques for prevention of treatment -Induced Ovarian ablation include better shielding of the ovaries from the damaging effects of radiation. This can be accomplished by implementing transposition procedures to move the ovaries outside of the area at risk. In addition knowledge of the precise three dimensional location of the ovaries allow the physician to limit the dose in these regions with novel beam arrangements, three dimensional conformal radiation therapy or Intensity modulated radiation Therapy. Prevention of the cytotoxic effects of the chemotherapy by administering a concurrent GnRHagonist (GnRHa) appears to be promising. In modern era of the improved antineoplastic age and highly conformal, three dimensional planned radiation therapy, the possibility of limiting the ovaries from the excess treatment-related toxicity in certain situations is becoming more of a reality.

\section{Treatment Methods}

\subsection{Follicle -Stimulating Hormone (FSH)}

FSH stimulates the growth and recruitment of immature ovarian follicles in the ovary. In early (small) antral follicles, FSH is the major survival factor that rescues the small antral follicles $(2-5 \mathrm{~mm}$ in diameter for humans)from apoptosis programmed death of the somatic cells of the follicle and oocyte .In addition , gonadotropin surge - attenuating factor produced by small follicles during the first half of the follicles phase also exerts a negative feedback on pulsatile luteinizing hormone (LH)secretion amplitude thus allowing a more favorable environment for follicle growth and preventing premature luteinization.

\subsection{Estradiol}

In the females, estradiol acts as a growth hormone for tissue of the reproductive organs, supporting the lining of the vagina, the cervical glands, the endometrium and the lining of the fallopian tubes. It enhances growth of the myometrium. Estradiol appears necessary to maintain oocytes in the ovary. During the menstrual cycle, estradiol produced by the growing follicle triggers, via a positive feedback system, the hypothalamicpituitary events that lead to the luteinizing hormone surge, inducing ovulation. The development of secondary sex characteristics in women is driven by estradiol. Estradiol produces breast development, and is responsible for changes in the body shape, affecting bones, joints and fat deposition.

\subsection{Preliminary Design: (concepts)}

Premenopausal women treated for Hodgkin and non Hodgkin Lymphoma were given three to eight cycles of chemotherapy or high dose therapy. At the time of random experiment it was observed that FSH and estradiol levels were less. Eligible patients were randomly assigned to receive chemotherapy and high dose therapy. The treatment was initiated 10days before the start of chemotherapy if possible. FSH and estradiol levels were measured in each center before the start of treatment, after 10 days ,after 3 months and at the end of 
the chemotherapy treatment .During the follow up period ,FSH and estradiol levels were evaluated at 3,6 and 12 months. According to the oncologists recommendation the patients were allowed to take oral contraception or other hormonal replacement therapy during the follow-up period. However FSH and estradiol levels were tested at least 10 days after the suspension of the medication. For the remaining patients the blood tests were preferentially performed at the beginning of the menstrual cycle. Treatment -related adverse effects were monitored at each visit.

\subsection{Ovarian Function Follow up}

\section{Indentations and Equations}

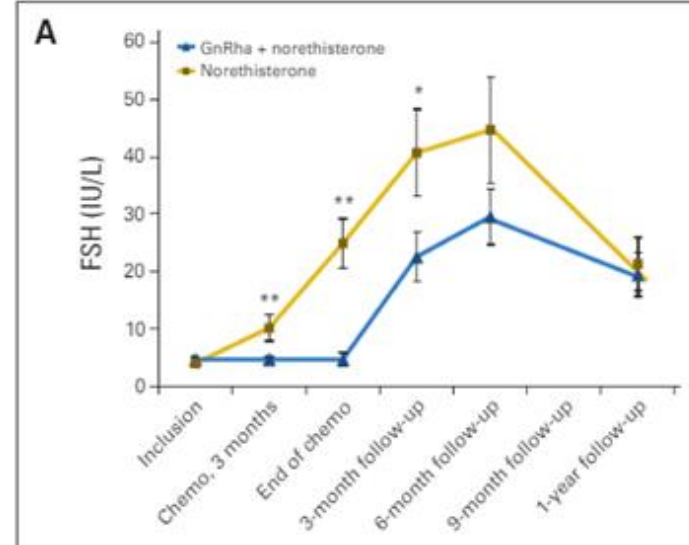

Fig. A: Follicle stimulating hormone (FSH)

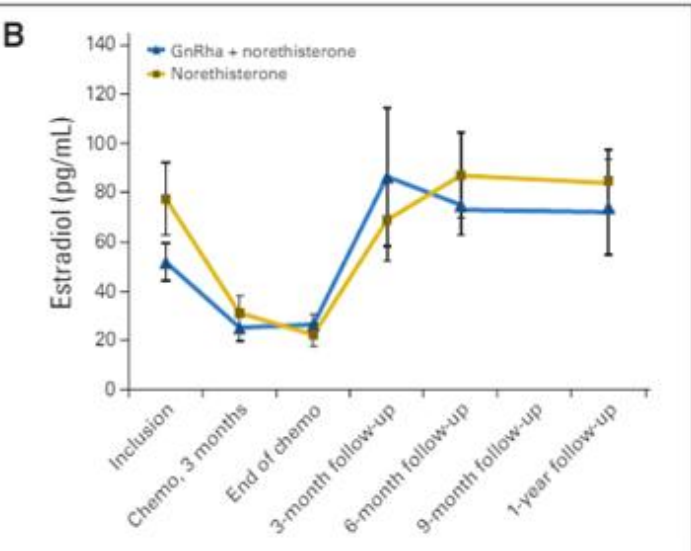

Fig. B: Estradiol values

The above Time points considered at inclusion, after 3 months of chemotherapy, at completion of chemotherapy and at 3,6 and 12 months of follow up with GnRHa(Gonadotropin releasing hormone agonist)

\subsection{FSH and Estradiol Levels for GnRHa Group and Control Group}

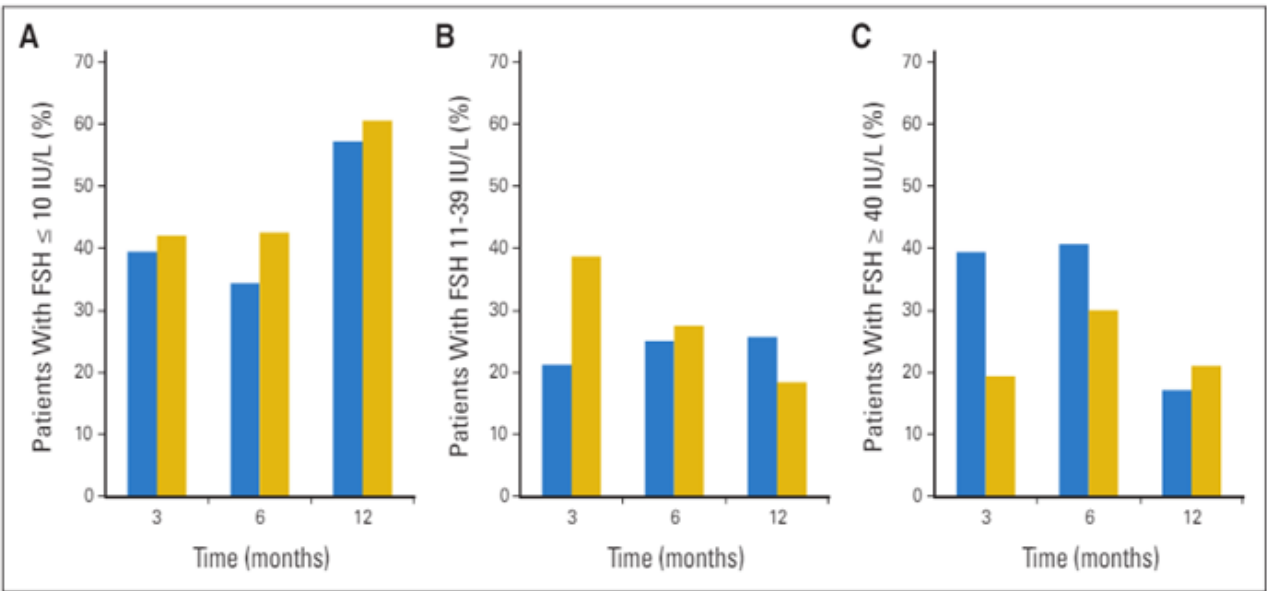

Percentage of women with follicle stimulating hormone (FSH) and estradiol values.

Fig. A: $\leq 10 \mathrm{IU} / \mathrm{L}$

Fig. B: between 11 and 39 IU/L

Fig. C: $\geq 40 \mathrm{IU} / \mathrm{L}$

The above figure shows the Gonadotropin releasing hormone agonist group(GOLD)and control group (BLUE) at different follow up times (3,6 and 12 months)

\subsection{Characterization of Model for Generating Bivariate Life Distributions using Reversed Hazard Rates}

Let us denote by $\mathrm{X}=\left(\mathrm{X}_{1}, \mathrm{X}_{2}\right)$ two nonnegative component lives, $\mathrm{X}_{1}$ and $\mathrm{X}_{2}$. Let $\mathrm{Fi}$ (xi) be the marginal distribution function of $\mathrm{Xi}$, and fi (xi) be the density function, $\mathrm{i}=1,2$. By definition, the Reversed Hazard Rate (RHR), ai(xi), and the cumulative RHR., Ai(xi), are 


$$
\begin{gathered}
a_{i}\left(x_{i}\right)=\frac{f_{i}\left(x_{i}\right)}{F_{i}\left(x_{i}\right)}, \quad A_{i}\left(x_{i}\right)=\int_{x_{i}}^{\infty} a_{i}\left(u_{i}\right) d u_{i} . \\
F_{i}\left(x_{i}\right)=\exp \left\{-A_{i}\left(x_{i}\right)\right\}, \quad \frac{d}{d x_{i}} A_{i}\left(x_{i}\right)=-a_{i}\left(x_{i}\right), \quad i=1,2 .
\end{gathered}
$$

Making use of these marginal distributions of $\mathrm{X}_{1}$ and $\mathrm{X}_{2}$ let us present a Characterized Extension Model (CE Model) based on the following thoughts.

Basic thoughts: At the time of extending a univariate life distribution to a higher dimension one must retain the basic features of the original life distributions. Since the concept of RHR plays an important role in reliability theory, as may be seen from the works of Keilson and Sumita (1982), Shaked and Shanthikumar (1994), Sengupta and Nanda (1997), Block et al. (1998), and Chandra and Roy (2001), we would like to incorporate in the bivariate model the retention of univariate RHR structure. Thus, functional forms of al $\left(\mathrm{X}_{1}\right)$ and $\mathrm{a} 2\left(\mathrm{X}_{2}\right)$ are the two basic structures that one should retain in the bivariate system and this retention should logically be in terms of the corresponding bivariate RHRs. Let $F\left(X_{1}, X_{2}\right)$ be the distribution function of $X=\left(X_{1}\right.$, $\mathrm{X}_{2}$ ). We propose that the corresponding bivariate RHRs be defined by

$$
a_{i}\left(x_{1}, x_{2}\right)=-\frac{\delta}{\delta x_{i}} A\left(x_{1}, x_{2}\right), \quad i=1,2,
$$

Where $\mathrm{A}\left(\mathrm{X}_{1}, \mathrm{X}_{2}\right)=-\log \mathrm{F}\left(\mathrm{X}_{1}, \mathrm{X}_{2}\right)$ Is the bivariate cumulative RHR function.

THEOREM 1: Bivariate RHRs are locally proportional to the corresponding variate RHRs if the bivariate distribution function is of the form

$$
F\left(x_{1}, x_{2}\right)=F_{1}\left(x_{1}\right) F_{2}\left(x_{2}\right) \exp \left\{-\gamma\left(\log F_{1}\left(x_{1}\right)\right)\left(\log F_{2}\left(x_{2}\right)\right)\right\}
$$

For same $\gamma$

It may be noted from the above result that the form of the characterized model given at (5.5) can be used for bivariate extension of univariate distributions retaining univariate structure of RHRs. Let us examine the set of values of $\gamma$ for which (5.5) is a proper distribution function. The following result provides an answer to this problem by specifying the range of values for $\gamma$.

THEOREM 2: The distribution function $\mathrm{F}(\mathrm{x} 1, \mathrm{x} 2)$ defined through the CE Model at 5.5 is defined if and only if $0 \leq \gamma \leq 1$. The corresponding joint density function is given by

$$
\begin{aligned}
f\left(x_{1}, x_{2}\right)= & F_{1}\left(x_{1}\right) F_{2}\left(x_{2}\right) a_{1}\left(x_{1}\right) a_{2}\left(x_{2}\right)\left[\left\{1+\gamma A_{1}\left(x_{1}\right)\right\}\left\{1+\gamma A_{2}\left(x_{2}\right)\right\}-\gamma\right] \\
& \cdot \exp \left[-\gamma\left(\log F_{1}\left(x_{1}\right)\right)\left(\log F_{2}\left(x_{2}\right)\right)\right] .
\end{aligned}
$$

Regarding the specific applications of the CE model we consider bivariate generalization of inverse exponential distribution, which is of special importance in RHR analysis. The distribution function of an inverse exponential variable, $X$, is $F(x)=\exp (-\gamma / x)$ so that $(1 / X)$ follows an exponential distributions, we note that cumulative RHRs are $\chi 1 / x 1$ and $\chi 1 / x 2$. Then, from theorem 1 we get the Bivariate Inverse Exponential Distribution as following

$$
F\left(x_{1}, x_{2}\right)=\exp \left\{\frac{-\lambda_{1}}{x_{1}}-\frac{\lambda_{2}}{x_{2}}-\gamma \frac{\lambda_{1}}{x_{1}} \frac{\lambda_{2}}{x_{2}}\right\}
$$

\section{Figures and Tables}

\section{Mathematical Interpretation}

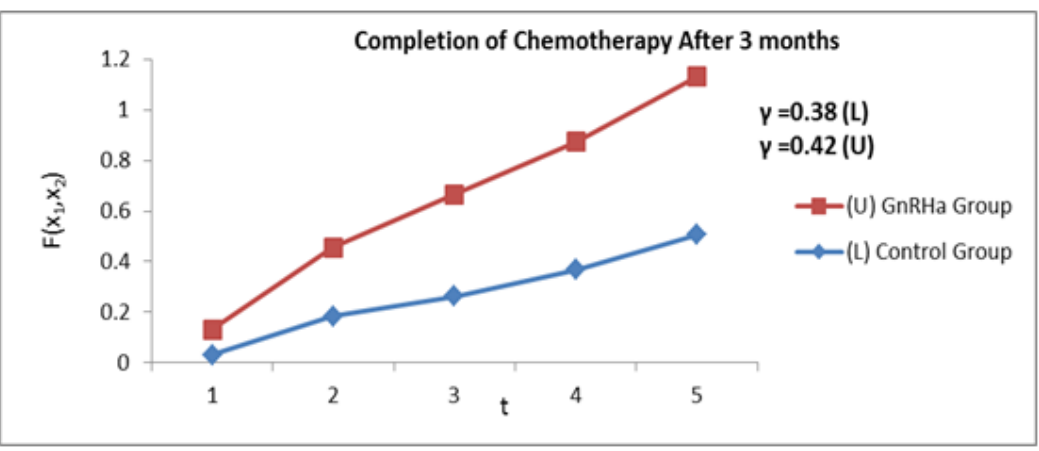



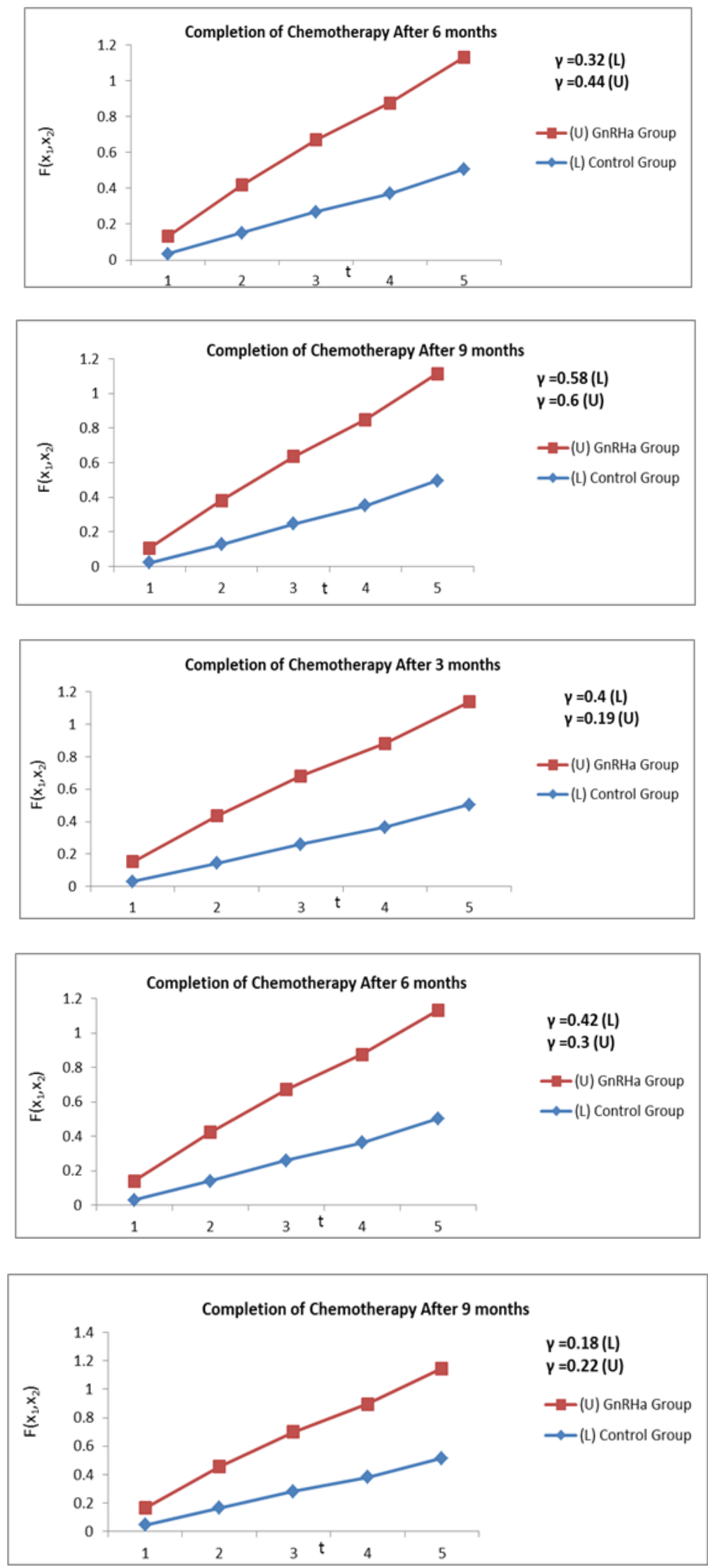

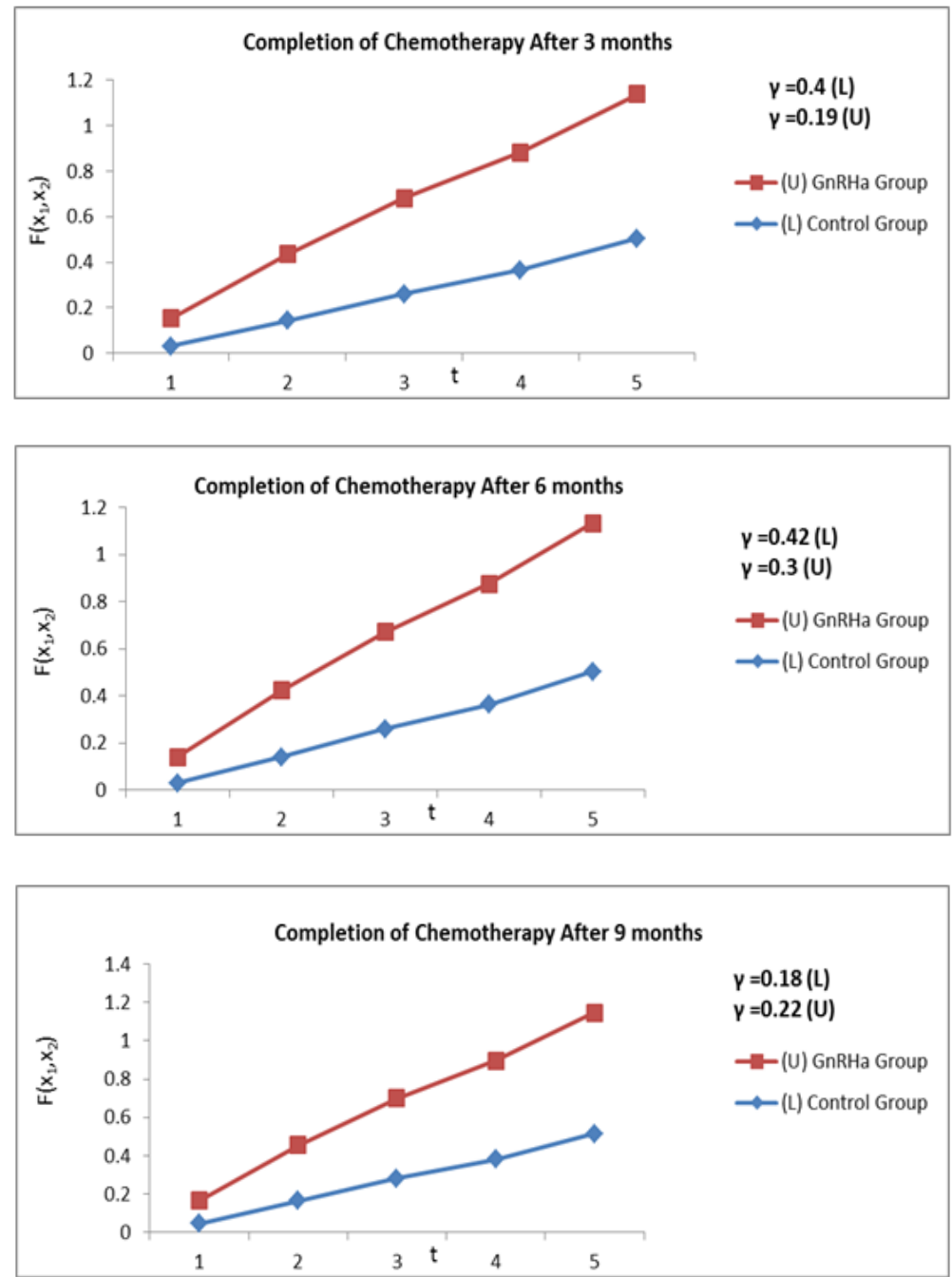

\section{Conclusion}

a. Mathematical Conclusion

- Combined effects of FSH and estradiol for GnRHa group is higher than the corresponding control group. First group of three figures shows the combined effects of FSH and estradiol in the GnRHa group are higher than the corresponding control group for different $\gamma$ values like $\gamma=0.38,0.42 ; \gamma=0.32,0.44 ; \gamma=0.58,0.6$.

- Second group of three figures also shows the combined effects of FSH and estradiol in the GnRHa group are higher than the corresponding control group for different $\gamma$ values like $\gamma=0.2,0.38 ; \gamma=0.25,0.25$; $\gamma=0.27,0.18$

- Third group of three figures shows the combined effects of FSH and estradiol in the GnRHa group are higher than the corresponding GnRHa group for different $\gamma$ values like $\gamma=0.4,0.19 ; \gamma=0.42,0.3 ; \gamma=0.18,0.22$.

There is a vast difference between GnRH agonist and control group in the third set of figures if we compare it with the first set and second set of figures.

\section{b. Medical Conclusion drawn from Mathematical Model}

- Medical conclusion suggests that a long term benefit of GnRHa on fertility of patients who spontaneously experience restoration of ovarian function. Mathematical model in the form of characterized model for bivariate extension gives a proper distribution function i.e. $\left(\mathrm{F}\left(\mathrm{X}_{1}, \mathrm{X}_{2}\right)\right.$ clearly seen in the mathematical graphs) for a given set of different values of $\gamma$. From the mathematical model it is clearly seen that combined effects of FSH and estradiol in the GnRHa group are higher than the corresponding control group. This gives a good conclusion to the medical professionals to measure the level of FSH in each chemotherapy treatment with GnRHa after the period of 3,6 and 12 months follow-up. 


\section{References}

[1] Woodruff T K, Preserving fertility during cancer treatment, NAT MED 15,2009,1124-1125.

[2] Peccatori F, Demeestere I, GnRH analogue for chemotherapy-induced ovarian damage; Too early to say?Fertil Steril,92;2009,e33.

[3] Beck-Fruchter R, Weiss A, Shalev E, GnRH agonist therapy as a ovarian protectants in female patients undergoing chemotherapy; a review of clinical data. Hum Reprod update 14,2008, 553-561.

[4] Thomas P Curran, Miguel Ángel Prieto Lage, Yvonne Anders, José Antonio Vázquez Álvarez, Miguel Anxo Murado García ,Development of bivariate mathematical model to characterize simultaneously the dose-time responses of pro-oxidant agents, Paper number 131620326, 2013.

[5] Pereyra Pacheco B, Mendez Ribas JM, Milone J, Use of GnRH analogs for functional protection of the ovaries and the preservation of fertility during cancer treatment in adolesents: a preliminary report, Gynecol Oncol 81,2001,391-397.

[6] Huser M, Crha I, Ventruba P, Prevention of ovarian function damage by a Gn RH analog during chemotherapy in Hodgkin lymphoma patients, Hum Reprod 23, 2008, 863-868.

[7] Rose,C. and smith,M.D." The Bivariate Normal". \$6.4 A in Mathematical Statistics with Mathematica.New York: SpringerVerlag,2002, pp.216-226.

[8] Rose,C. and smith, M.D. "The Multivariate Normal Distribution". Mathematica J.6,1996,32-37.

[9] Samuel Kotz, N. Balakrishnan, Norman L. Johnson, Continuous Multivariate Distributions, Volume 1, Models and Applications, 2nd Edition (Wiley). 